\title{
OFDM TURBO DECODULATION WITH EXIT OPTIMIZED BIT LOADING AND SIGNAL CONSTELLATIONS
}

\author{
Helge Lüders, Benedikt Eschbach, Laurent Schmalen, Peter Vary \\ Institute of Communication Systems and Data Processing (ind) \\ RWTH Aachen University, Germany \\ \{lueders|eschbach |schmalen|vary\} dind.rwth-aachen. de
}

\begin{abstract}
We propose the combination of Orthogonal Frequency Division Multiplexing (OFDM) and Turbo DeCodulation (TDeC) - a multiple Turbo process consisting of iterative demodulation and iterative source-channel decoding - for transmission of correlated source codec parameters over wireless frequency-selective broadband fading channels. As OFDM systems split up frequency-selective fading channels into orthogonal flat-fading channels, individual modulation signal constellations sets with different bit mapping rules can be assigned to subcarriers taking into account the iterative reception and decoding process. The superior performance of the proposed OFDM-TDeC system is demonstrated by analyzing the achieved residual bit error rate (BER) and parameter signal-to-noise ratio (SNR).
\end{abstract}

Index Terms - Turbo DeCodulation, Turbo Principle, BICM-ID, ISCD, OFDM

\section{INTRODUCTION}

With the discovery of Turbo codes [1] channel coding close to the Shannon limit has become possible with moderate computational complexity. In the past years the Turbo principle of exchanging extrinsic information between separate channel decoders has been extended to other components of the receiver. The iterative combination of demodulator, channel decoder, and source decoder with two iterative loops called Turbo DeCodulation (TDeC) [2] provides performances near the optimum performance theoretically attainable (OPTA) [3] in additive white Gaussian noise (AWGN) and flat Rayleigh fading environments. Orthogonal Frequency Division Multiplexing (OFDM) on the other hand has proven to be a modulation technique that provides high spectral efficiency while keeping the complexity at a low level for transmissions over frequency-selective broadband fading channels. For that reason OFDM was chosen for current and evolving wireless communication systems such as IEEE 802.16-2004 (WiMAX) or UMTS Long Term Evolution (LTE).

In order to also employ $\mathrm{TDeC}$ in time-varying frequencyselective fading environments with intersymbol-interference (ISI) we combine both concepts and prove the applicability of the resulting OFDM-TDeC by simulation. We believe, that this bidirectionally linking of application and physical layer components enables the kind of cross-layer optimization required by the next generation of mobile communication and can be applied wherever these components are physically co-located, e.g., in mobile devices.

This work has been supported by the l|lumic (Ultra High-Speed Mobile Information and Communication) research cluster.
In addition to the possibility of transmitting source coded data over frequency-selective multi-path channels with reasonable complexity, another benefit of the combination of OFDM and TDeC is the option to exploit the ability of OFDM systems to transform these channels into parallel flat fading channels. We introduce a strategy for individually assigning modulation signal constellation sets (SCS) and bit mapping rules to different subcarriers, thereby optimizing the convergence behavior of the inner iterative loop with respect to the residual bit error rate. This method dynamically adapts the transmitter and receiver to the current channel condition. The performance of the OFDM-TDeC system and its adapted version is analyzed and illustrated by means of extrinsic information transfer (EXIT) charts [4], residual bit error rate (BER), and parameter signal-to-noise ratio (SNR).

\section{THE OFDM TURBO DECODULATION SYSTEM}

In Fig. 1 the baseband model of the proposed OFDM Turbo DeCodulation system is depicted. We generally follow the system model for TDeC given in [2] but use a simplified notation to describe only the functionality relevant for this contribution. For a detailed description of the transmission and reception process the reader is referred to [2] and the literature given there.

Source codec parameters $u$ of a block $\mathbf{u}$ are quantized and mapped to unique bit patterns $\underline{v}$ individually representing quantizer reproduction levels $\bar{u}^{(\underline{v})}$. The concatenation of these bit patterns forms the data bit block $\mathbf{v}$. After outer interleaving with interleaver $\pi_{\text {out }}$ the resulting block $\tilde{\mathbf{v}}$ is encoded by a convolutional encoder to become the code bit block $\mathrm{x}$ which then is interleaved by the inner

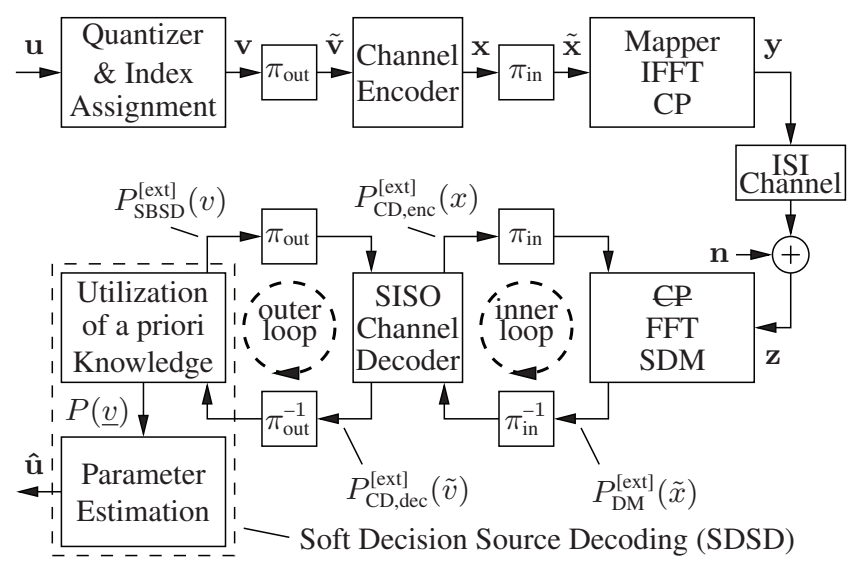

Fig. 1. Baseband model of the OFDM Turbo DeCodulation system. 
interleaver $\pi_{\text {in }}$.

Differing from [2], the interleaved block of coded bits $\tilde{\mathbf{x}}$ is then passed to an OFDM modulator where the block is first split into groups of bit patterns $\tilde{\mathbf{x}}_{m}$ of predefined lengths $I_{m}$ with $m \in[0, \ldots, M-1]$ representing the $M$ subcarriers of the OFDM system. Therefore, one OFDM symbol carries $I_{\Sigma} \doteq \sum_{m} I_{m}$ coded bits. If the size of the vector of coded bits $\tilde{\mathbf{x}}$ is not an integer multiple of $I_{\Sigma}$ the vector is zero-padded. The patterns $\tilde{\mathbf{x}}_{m}$ are subcarrier-wise mapped to complex modulation symbols $Y_{m}$ with $\mathrm{E}\left\{\left|Y_{m}\right|^{2}\right\}=1$ out of the signal constellation set (SCS) $\mathcal{Y}$ according to a subcarrierdependant mapping rule $\mu_{m}$ so that $Y_{m}=\mu_{m}\left(\tilde{\mathbf{x}}_{m}\right)$. In this work we restrict ourselves to bit patterns of size $I_{m} \in[2,3,4]$ with different signal constellation sets $\mathcal{Y}$ and mapping rules $\mu$. In the following the expression $\mathcal{Y}_{\mu}$ will refer to a specific combination of SCS and mapping rule. $M$ complex modulation symbols are considered as one OFDM symbol. A $M$-point inverse Fourier transform (IFFT) is performed on each OFDM symbol and a cyclic prefix (CP) is added. The resulting signal vector $\mathbf{y}$ consists of complex samples $y$. Taking into account the $\mathrm{CP}$ and a possibly large peak-to-average power ratio (PAPR) $y$ will be scaled to unit power on the long-term average, i.e., $\mathrm{E}\left\{|y|^{2}\right\}=1$. Note that signal vectors in the transform domain equivalent (before IFFT resp. after FFT) of the mapper/modulator resp. demodulator/demapper are indicated by upper case letters, while signal vectors in the time domain equivalent (after IFFT resp. before FFT) are indicated by lower case letters.

Throughout this work we are considering a frequency selective multi-path propagation channel causing intersymbol interference (ISI). This band limited ISI channel is modeled by a tapped delay line. One complex time variant coefficient $c_{k}(t)$ in each tap models the individual fading comprising the effects of the static attenuation, shadowing, and fast fading. The channel impulse response (CIR) $c(t, \tau)$ of length $L+1$ is given by:

$$
c(t, \tau)=\sum_{k=0}^{L} c_{k}(t) \delta(k T-\tau), \quad c_{k} \in \mathbb{C} .
$$

Despite the time-variant nature of the fading coefficients of each tap, we assume the channel to be constant for the duration of the transmission of at least one OFDM symbol. The channel is then described by a CIR vector consisting of its complex fading coefficients

$$
\mathbf{c}=\left[c_{0}, c_{1}, \ldots, c_{L}\right]
$$

which are sufficiently well estimated at the receiver. Finally, complex additive white Gaussian noise (AWGN) $\mathbf{n}$ with a known power spectral density of $\sigma_{n}^{2}=N_{0}$ is applied.

After cyclic prefix removal and fast Fourier transform (FFT) the received complex symbols of each subcarrier $Z_{m}$ are given as

$$
Z_{m}=C_{m} \cdot Y_{m}+N_{m}, m \in\{0, \ldots, M-1\}
$$

with $N_{m}$ denoting Fourier transformed noise samples and $C_{m}$ denoting the samples of the transfer function of length $M$ of the channel c. It follows that after division by $C_{m}$ (equalization) the subchannels can be regarded as individual (though correlated) AWGN channels with noise power

$$
\sigma_{n, m}^{2} \doteq \mathrm{E}\left\{\left(\frac{N_{m}}{C_{m}}\right)^{2}\right\}=\frac{\sigma_{n}^{2}}{\left|C_{m}\right|^{2}} .
$$

As shown in Fig 1, the receiver consists of two iterative loops. The inner loop corresponds to a bit-interleaved coded modulation with iterative decoding (BICM-ID) system [5] where the soft demapper (SDM) and the soft input soft output (SISO) channel decoder (CD) exchange extrinsic information on the encoded bits in form of extrinsic probabilities $P_{\mathrm{DM}}^{[\mathrm{ext}]}(\tilde{x})$ and $P_{\mathrm{CD} \text {,enc }}^{\text {[ext] }}(x)$ respectively [2].
The outer loop is an iterative source channel decoding (ISCD) system [6], [7]. After the final outer iteration parameter-oriented a posteriori knowledge $P(\underline{v})$ is determined for each quantizer reproduction level $\underline{v}$. Each parameter $\hat{u}$ is then estimated by soft decision source decoding (SDSD) employing these probabilities $P(\underline{v})$ as weighting factors for a summation of all possible quantizer reproduction levels $\bar{u}^{(\underline{v})}$ :

$$
\hat{u}=\sum_{\underline{v}} \bar{u}^{(\underline{v})} \cdot P(\underline{v}) .
$$

Again, we refer to [2] for a more detailed description of the employed iterative decoding stages.

\section{EXIT OPTIMIZATION OF THE BICM-ID STAGE}

The optimized single carrier TDeC system proposed in [2] employs 8PSK with mixed signal space mapping [5] in combination with an optimized quantizer bit mapping of the source encoder. For the BICM-ID stage alone however, semi-set partitioning (SSP) [5] is the optimum mapping rule $\mu$ as it provides the lowest error-floor possible for 8PSK. Set partitioning (SP) on the other hand achieves an earlier water-fall for low channel qualities but yields a higher error-floor when compared to SSP. For OFDM systems it is known that individual signal constellation sets (SCS) on each subcarrier which depend on the current channel characteristics can maximize the throughput or minimize the BER, or power consumption of the system [8]. This concept is known as bit/power loading or water-filling.

Our goal is to minimize the residual BER of the BICM-ID stage while keeping a constant overall bit rate and an average constant transmit power on all subcarriers. To take into account the iterative behavior of BICM-ID, the EXIT characteristic of the SDM is considered which describes the amount of extrinsic information $\mathcal{I}_{\mathrm{DM}}^{[\mathrm{ext}]}$ that is created by the soft demodulator (SDM) at a certain channel quality from a certain amount of a priori information $\mathcal{I}_{\mathrm{DM}}^{[\text {apri] }}$ provided by the SISO CD: $\mathcal{I}_{\mathrm{DM}}^{[\mathrm{ext}]}=\mathcal{T}_{\mathrm{DM}}\left(\mathcal{I}_{\mathrm{DM}}^{[\mathrm{apri}]}, N_{0}\right)$. $\mathcal{I}_{\mathrm{DM}}^{[\mathrm{apri}]}=f\left(P_{\mathrm{CD}, \text { enc }}^{[\mathrm{ext}]}\right)$ and $\mathcal{I}_{\mathrm{DM}}^{[\mathrm{ext}]}=f\left(P_{\mathrm{DM}}^{[\mathrm{ext}]}\right)$ are functions of the extrinsic probabilities exchanged in the BICM-ID stage (see Fig. 1). A so-called decoding tunnel has to exist between the EXIT characteristic of the SDM and the inverse EXIT characteristic of the SISO channel decoder $\mathcal{I}_{\mathrm{DM}}^{\text {[ext] }}=\mathcal{T}_{\mathrm{CD}}^{-1}\left(\mathcal{I}_{\mathrm{DM}}^{\text {[apri] }}\right)$. The constraint is $\mathcal{T}_{\mathrm{DM}}\left(\mathcal{I}_{\mathrm{DM}}^{\text {[apri] }}, N_{0}\right)>\mathcal{T}_{\mathrm{CD}}^{-1}\left(\mathcal{I}_{\mathrm{DM}}^{[\mathrm{apri}]}\right)$ for the largest possible range of $\mathcal{I}_{\mathrm{DM}}^{\text {[apri] }}$, as the residual BER will decrease with an increasing range of $\mathcal{I}_{\mathrm{DM}}^{\text {[apri] }}$ [9]. All SDM characteristics $\mathcal{T}_{\mathrm{DM}}$ are monotonically increasing over $\mathcal{I}_{\mathrm{DM}}^{[\mathrm{apri}]}$ but their individual progressions heavily depend on the chosen $\mathcal{Y}_{\mu}$ as well as on the channel quality. It has been shown in [10] that the resulting $\mathcal{T}_{\mathrm{DM}}$ of an OFDM system can be calculated as weighted sum of the single SDM characteristics of each subcarrier depending on the CIR $\mathbf{c}$ and the AWGN power $N_{0}$. Employing individual $\mathcal{Y}_{\mu}$ on the subcarriers will thus produce different SDM characteristics $\mathcal{T}_{\mathrm{DM}}$ for the BICM-ID stage. Therefore, the goal is to find the assignment of $\mathcal{Y}_{\mu}$ to the subcarriers that allows for the maximum iterative gain, i.e., for a decoding tunnel of maximum length and width. The optimization problem is formulated as:

$$
\max _{\mathcal{I}_{\mathrm{DM}}}\left(\mathcal{I}_{\max }^{[\mathrm{apri}]} \mid \mathcal{T}_{\mathrm{DM}}(\mathcal{I})>\mathcal{T}_{\mathrm{CD}}^{-1}(\mathcal{I})+\tau \text { and } 0 \leq \mathcal{I} \leq \mathcal{I}_{\max }^{[\mathrm{apri}]}\right)
$$

with threshold $\tau$ ensuring the convergence of the BICM-ID stage for limited block lengths and numbers of iterations.

A full search for a large number of subcarriers and combinations $\mathcal{Y}_{\mu}$ would be far too complex to perform once per period the channel is considered time-invariant, e.g., one OFDM symbol. Thus, we 
propose the following two-step approach to assign constellation and mapping sets to subcarriers:

1) Assign a bit pattern length $I_{m}$ (rate) to each subcarrier $m$ For bit loading we define the subcarrier specific channel quality $\mathrm{SNR}_{m}$ and, motivated by [8], the optimization criterion $Q$ :

$$
\begin{aligned}
\mathrm{SNR}_{m} & \doteq-10 \log _{10}\left(\sigma_{n, m}^{2}\right) \\
Q & \doteq \sum_{m=0}^{M-1} \frac{\mathrm{SNR}_{m}}{I_{m}} \rightarrow \min ,
\end{aligned}
$$

where $Q$ has to be minimized while keeping a constant overall bit rate of, e.g., $I_{\Sigma}=3 \cdot M$ bit per OFDM symbol. This is achieved by Alg. 1: $I_{m}=3$ bits are assigned to subcarriers with medium $\mathrm{SNR}_{m}$ while $I_{m}=2$ bit and $I_{m}=4$ bit are assigned pairwise to subcarriers with low and high $\mathrm{SNR}_{m}$ respectively.

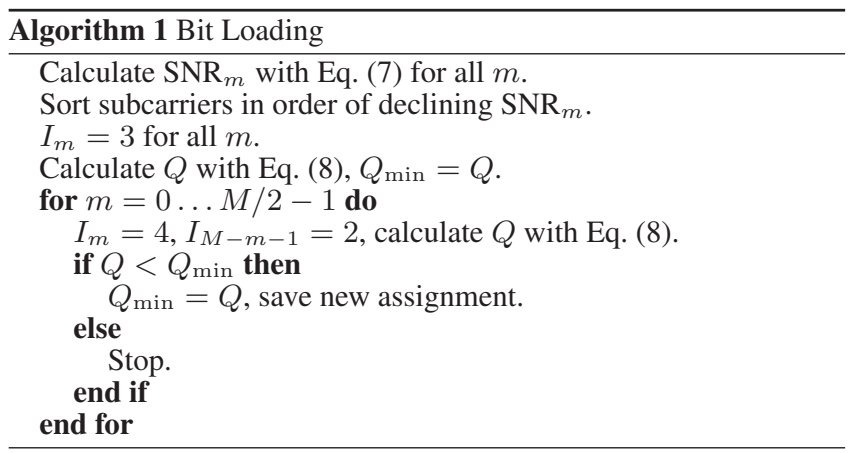

\section{2) Assign best $\mathcal{Y}_{\mu}$}

In the second step a signal constellation set and mapping rule $\mathcal{Y}_{\mu}$ that maximizes the length of the decoding tunnel (cf. Eq. (6)) is assigned consecutively to each subcarrier following Alg. 2.

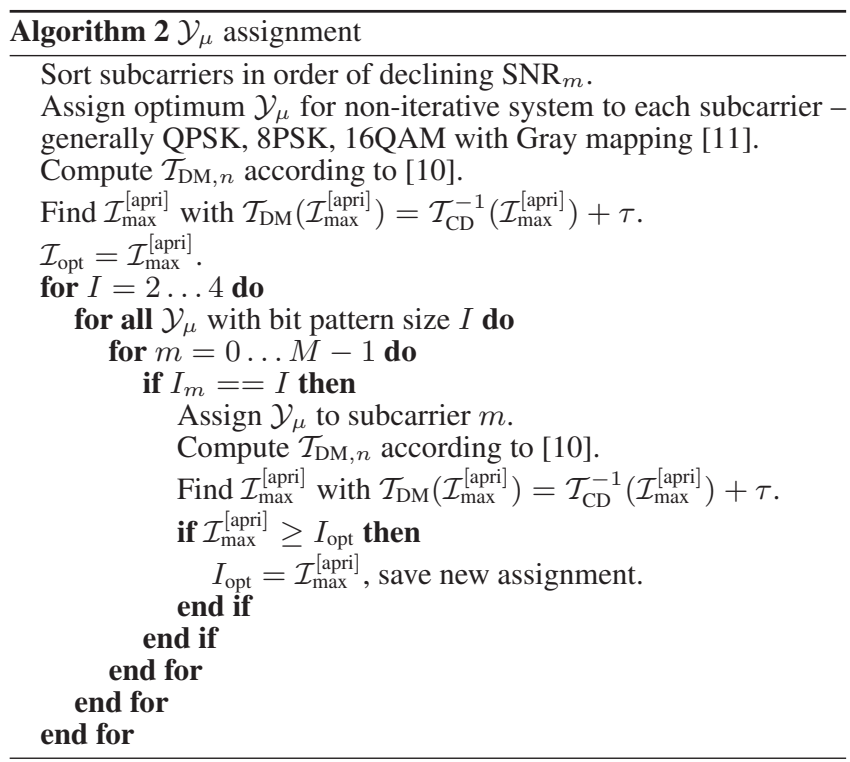

\section{SIMULATION EXAMPLES}

The applicability of the OFDM-TDeC system introduced in Sec. 2 and the performance gains achieved with the optimization of the
BICM-ID stage proposed in Sec. 3 are demonstrated by simulation. An OFDM modulator with $M=256$ subcarriers is used and an ISI channel is exemplarily chosen as real FIR with CIR $\mathbf{c} \sim$ $[1.0,0.0,0.5,0.2,0.1]$. Consequently the length of the cyclic prefix is $L=4$.

\subsection{BICM-ID optimization}

For the EXIT optimization of the BICM-ID stage we investigated the transmission of data blocks each containing 200000 bits. The bits were encoded using a rate $1 / 2$ feed-forward convolutional encoder with zero termination, constraint length 4 , and octal generator polynomials $G_{0}=\{17\}_{8}$ and $G_{1}=\{15\}_{8} .9$ possible $\mathcal{Y}_{\mu}$ have been selected for the optimization of the mapper which are summarized in Tab. 1. These $\mathcal{Y}_{\mu}$ have been chosen regarding the variety of progressions of their SDM characteristics.

\begin{tabular}{|c|c|c|}
\hline Bits per symbol $I$ & SCS $\mathcal{Y}$ & Mapping rule $\mu$ \\
\hline$I=2$ & QPSK & Gray, Anti-Gray \\
\hline$I=3$ & 8PSK & Gray, SP, SSP \\
\hline \multirow{3}{*}{$I=4$} & 16QAM & Gray, A1 [12] \\
\cline { 2 - 3 } & 16QAMw [13] & optimized [13] \\
\cline { 2 - 3 } & 16PSK & Ray [12] \\
\hline
\end{tabular}

Table 1. $\mathcal{Y}_{\mu}$ for EXIT optimization of the BICM-ID stage.

The EXIT chart resulting from the optimization is depicted in Fig. 2. It can be seen that for the exemplary target channel quality of $E_{S} / N_{0}=5 \mathrm{~dB}$ no decoding tunnel exists for 8PSK-SSP. For the $\mathcal{Y}_{\mu}$ assignment optimized according to Sec. 3 a tunnel exists enabling the system to converge already after 10 iterations (stair steps of the decoding trajectory). Furthermore, the SDM characteristic of the optimized system reaches an even higher value of $\mathcal{I}_{\mathrm{DM}}^{[\mathrm{ext}]}$ for the error-free feedback $(\mathrm{EFF})$ case at $\mathcal{T}_{\mathrm{DM}}\left(\mathcal{I}_{\mathrm{DM}}^{[\mathrm{apri}]}=1\right)$. Note that the area property of the EXIT chart stating that the area beneath the SDM characteristic corresponds to the capacity of the channel for the regarded signal constellation sets is not harmed: the superposition of SCS with different numbers of bits $I_{m}$ can lead to an increased capacity compared to a set up using, e.g., only SCS with $I_{m}=3$ bit for all subcarriers. These findings are confirmed by the residual bit error rates given in Fig. 3: For the optimized assignment the waterfall region starts at the optimization point of $E_{S} / N_{0}=5 \mathrm{~dB}$, about $0.5 \mathrm{~dB}$ earlier compared to 8PSK-SSP and converges into an errorfloor which is about half a decade lower than this of 8PSK-SSP.

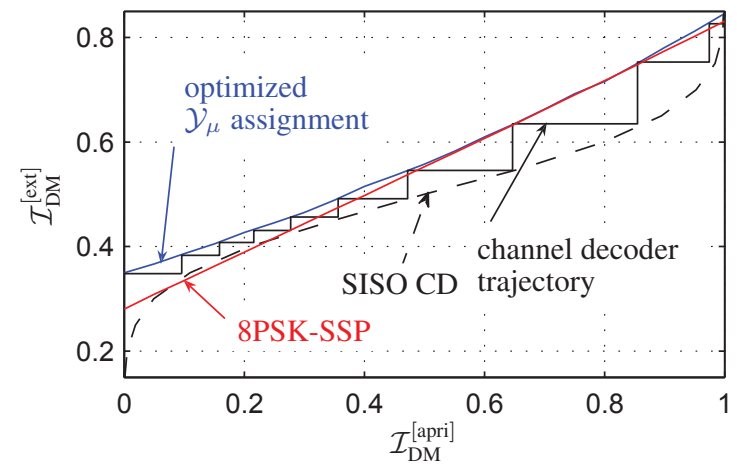

Fig. 2. EXIT charts for BICM-ID at $E_{S} / N_{0}=5 \mathrm{~dB}$. 


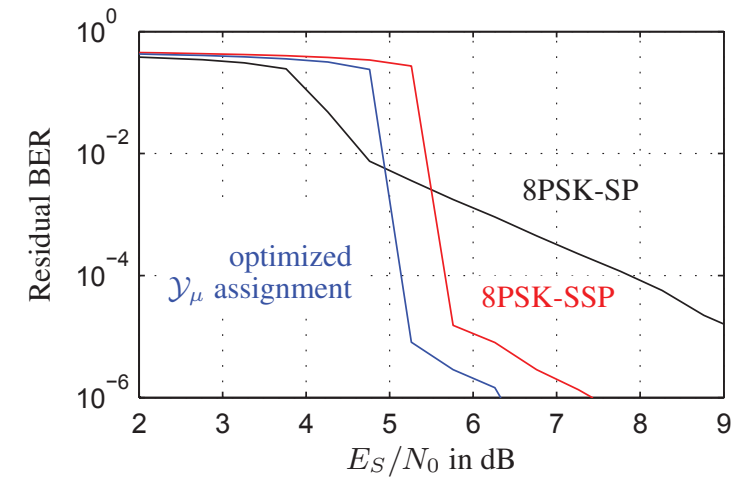

Fig. 3. Residual BER over $E_{S} / N_{0}$ after BICM-ID stage (10 BICMID iterations).

\subsection{OFDM-TDeC performance}

For the OFDM-TDeC the system parameters of the ISCD stage (codec parameter correlation, quantizers, quantizer bit mappings, ISCD iterations, etc.) have been chosen according to [2]. Parameter blocks $\mathbf{u}$ each containing 250 source codec parameters are transmitted and 10 inner loop iterations are executed. After estimation of the parameters $\hat{u}$ the parameter SNR $\mathcal{P}$ is calculated by $\mathcal{P}=E\left\{u^{2} /(u-\hat{u})^{2}\right\}$ as a measure of quality which, due to the selected quantizers, is upper bounded by $\mathcal{P}_{\max } \approx 14.6 \mathrm{~dB}$ [3].

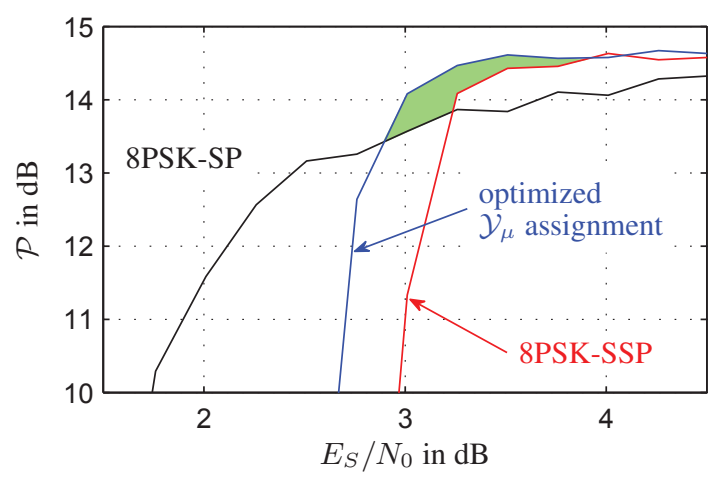

Fig. 4. Parameter SNR over $E_{S} / N_{0}$ for OFDM-TDeC (10 BICM-ID iterations).

Figure 4 illustrates the simulation results for a TDeC system using 8PSK-SSP, 8PSK-SP, and an $\mathcal{Y}_{\mu}$ assignment optimized according to Sec. 3. It can be seen that a good performance concerning $\mathcal{P}$ is achieved for very low channel qualities of $E_{S} / N_{0} \approx 3 \mathrm{~dB}$ for all depicted cases which proves the applicability of OFDM-TDeC for frequency-selective ISI channels. Furthermore, the system with optimized $\mathcal{Y}_{\mu}$ assignment outperforms 8PSK-SSP concerning $\mathcal{P}$. 8PSKSP on the other hand outperforms both other depicted systems for very low channel qualities with the trade-off of reaching $\mathcal{P}_{\max }$ only for very high channel qualities. The corresponding gain of the optimized system is illustrated by the marked area in Fig. 4.

\section{CONCLUSIONS}

With OFDM-TDeC we introduced the combination of OFDM modulation with Turbo DeCodulation and demonstrated its applicability to time-varying frequency-selective broadband fading channels. Additionally, a strategy for assigning symbol constellation sets and mapping rules to subcarriers has been proposed which optimizes the BICM-ID stage of the OFDM-TDeC system with respect to the residual BER. The BER performance gains are illustrated in Fig. 3: An earlier waterfall as well as a lower error floor can be recognized in comparison to the optimum system with fixed symbol constellation sets and mapping rule. The gain in transmission quality given by the parameter SNR confirms these findings.

\section{ACKNOWLEDGMENT}

The authors wish to thank Thorsten Clevorn for valuable comments and fruitful discussions.

\section{REFERENCES}

[1] C. Berrou, A. Glavieux, and P. Thitimajshima, "Near Shannon limit error-correcting coding and decoding: Turbo-codes (1)," in IEEE International Conference on Communications (ICC), Geneva, Switzerland, May 1993.

[2] T. Clevorn, J. Brauers, M. Adrat, and P. Vary, "Turbo DeCodulation: Iterative Combined Demodulation and Source-Channel Decoding," IEEE Communications Letters, vol. 9, no. 9, pp. 820-822, Sept. 2005.

[3] T. Clevorn, L. Schmalen, P. Vary, and M. Adrat, "On The Optimum Performance Theoretically Attainable for Scalarly Quantized Correlated Sources," in Proceedings of International Symposium on Information Theory and its Applications (ISITA), Seoul, Korea, Oct. 2006.

[4] S. ten Brink, "Designing Iterative Decoding Schemes with the Extrinsic Information Transfer Chart," AEÜ Int. J. Electron. Commun., pp. 389 - 398, Nov. 2000.

[5] X. Li and J. A. Ritcey, "Bit-Interleaved Coded Modulation with Iterative Decoding," IEEE Communications Letters, vol. 1, no. 6, pp. 169 - 171, Nov. 1997.

[6] M. Adrat, P. Vary, and J. Spittka, "Iterative Source-Channel Decoder Using Extrinsic Information from Softbit-Source Decoding," in IEEE International Conference on Acoustics, Speech, and Signal Processing (ICASSP), Salt Lake City, USA, May 2001.

[7] N. Görtz, "On the Iterative Approximation of Optimal Joint Source-Channel Decoding," IEEE Journal on Selected Areas in Communications, vol. 19, no. 9, pp. 1662-1670, Sept. 2001.

[8] C. Assimakopoulos and F. N. Pavlidou, "Unified models for adaptive OFDM systems when QAM or PSK modulation is applied," European Transactions on Telecommunications, vol. 18, no. 7, pp. 777-790, Feb. 2007.

[9] T. Clevorn, S. Godtmann, and P. Vary, "BER prediction using EXIT charts for BICM with iterative decoding," IEEE Communications Letters, pp. 49-51, Jan. 2006.

[10] H. Lüders, L. Schmalen, and P. Vary, "Approximating EXIT Characteristics of Soft Demodulators in OFDM BICM-ID Systems for Time Variant Frequency Selective Fading Channels," in Proceedings of International ITG Conference on Source and Channel Coding, Ulm, Germany, Jan. 2008.

[11] G. Caire, G. Taricco, and E. Biglieri, "Bit-Interleaved Coded Modulation," IEEE Transactions on Information Theory, pp. 927-946, May 1998.

[12] T. Clevorn, S. Godtmann, and P. Vary, "PSK versus QAM for Iterative Decoding of Bit-Interleaved Coded Modulation," in Proceedings of IEEE Global Telecommunications Conference (GLOBECOM), Dec. 2004.

[13] N. S. Muhammad and J. Speidel, "Joint optimization of signal constellation bit labeling for bit-interleaved coded modulation with iterative decoding," IEEE Communications Letters, vol. 9, no. 9, pp. 775-777, Sept. 2005. 